University of Wollongong

Research Online

Faculty of Informatics - Papers (Archive)

Faculty of Engineering and Information

Sciences

19-10-2003

\title{
Lie analysis of the Webster horn equation with application to audio object recognition
}

Tania M. Barrett

University of Wollongong, tbarrettt@uow.edu.au

Ian S. Burnett

University of Wollongong, ianb@uow.edu.au

Jason Lukasiak

University of Wollongong, j101@ouw.edu.au

Follow this and additional works at: https://ro.uow.edu.au/infopapers

Part of the Physical Sciences and Mathematics Commons

\section{Recommended Citation}

Barrett, Tania M.; Burnett, lan S.; and Lukasiak, Jason: Lie analysis of the Webster horn equation with application to audio object recognition 2003.

https://ro.uow.edu.au/infopapers/41

Research Online is the open access institutional repository for the University of Wollongong. For further information contact the UOW Library: research-pubs@uow.edu.au 


\title{
Lie analysis of the Webster horn equation with application to audio object recognition
}

\author{
Abstract \\ The Webster horn equation describes the pressure wave in a duct of slowly varying cross section. We \\ discuss symmetry reductions and exact solutions of the Webster horn equation using the classical Lie \\ method of infinitesimals. The particular case of the exponential horn is examined and a complete set of \\ reductions and solutions is formulated. The generation of a complete set of solutions using Lie analysis \\ produces a set of group transformations. Particular attention is given to a new solution found, which \\ contains an exponentially decaying Bessel function. The use of these group transformations as a tool for \\ audio object recognition is also explored. Results indicate that the decaying Bessel function solution \\ provides a particularly useful insight into exponential horn object recognition. Practical results are \\ presented which indicate the group transformations offer an exciting new mechanism for identifying a \\ specific audio object in a mixed audio scene.

\section{Disciplines} \\ Physical Sciences and Mathematics

\section{Publication Details} \\ This article was originally published as: Barrett, TM, Burnett, I \& Lukasiak, J, Lie Analysis of the Webster \\ Horn Equation with Application to Audio Object Recognition, 2003 IEEE Workshop on Applications of \\ Signal Processing to Audio and Acoustics, 19-22 October 2003, 217-220. Copyright IEEE 2003.
}




\title{
LIE ANALYSIS OF THE WEBSTER HORN EQUATION WITH APPLICATION TO AUDIO OBJECT RECOGNITION
}

\author{
Tania M Barrett, Ian S Burnett, Jason Lukasiak \\ Whisper Labs, School of Electrical, Computer and Telecommunications Engineering, University of \\ Wollongong, Wollongong, NSW, Australia \\ Email: taniadst.elec.uow.edu.au
}

\begin{abstract}
In this paper we discuss symmetry reductions and exact solutions of the Webster Horn Equation using the classical.Lie method of infinitesimals. The particular case of the exponential horn is examined and a complete set of reductions and solutions is formulated. The generation of a complete set of solutions using Lie analysis produces a set of group transformations. Particular attention is given to a new solution found, which contains an exponentially decaying Bessel function. The use of these group transformations as a tool for audio object recognition is also explored. Results indicate that the decaying Bessel function solution provides a particularly useful insight into exponential hom object recognition. Practical results are presented which indicate the group transformations offer an exciting new mechanism for identifying a specific audio object in a mixed audio scene.
\end{abstract}

\section{INTRODUCTION}

Lie analysis methods are used throughout Mathematics as a technique for finding a complete set of solutions to partial and ordinary differential equations [1], [2]. Recently these methods have also found application as an object recognition tool in twodimensional subspaces such as image processing [3], [4], with a notable degree of success. In this paper both of these applications are applied to a mono audio signal as described by the Webster Hom Equation.

A brief outline of the theory of Lie analysis is given in Section 2. In Section 3 the details of the application of Lie analysis to the Webster Horn Equation, establishing a complete set of group transformations and solutions is provided. Section 4 explores the use of applying the group transformations found in Section 3 to a mono audio signal mixture, which contains objects satisfying the Webster Horn Equation.

The Webster Horn Equation describes the pressure wave in a duct of slowly varying cross section. Methods for its derivation can be found in Blackstock [5], Putland [6] and Rienstra [7]. It is given by the following partial differential equation:

$$
p_{n}=c^{2}\left((\ln S(x))_{x} p_{x}+p_{x x}\right)
$$

where $c$ is the speed of sound, $S(x)$ is the surface area, and $p(x, t)$ is the pressure of the wave front. Subscript notation is used to denote partial differentiation. The case when $S(x)$ has an exponential profile holds particular interest in acoustic engineering, especially loudspeaker technology. In this instance we have $S(x)=S_{0} e^{m x}$ where $m$ is a flare constant. Substitution into our governing equation (1) results in the exponential Webster Horn Equation:

$$
p_{t t}=c^{2}\left(m p_{x}+p_{x x}\right)
$$

Most commonly the solution is given as a time-harmonic wave function, [5], [8]:

$$
p=A e^{j(a r-k x)} \text { with } k= \pm \frac{\omega}{c} \sqrt{1-\left(\frac{m c}{2 \omega}\right)^{2}}-j \frac{m}{2}(3),
$$

where $\omega$ is radian frequency.

\section{LIE GROUP ANALYSIS}

This section gives a brief overview of the ideas of Lie Symmetry methods for differential equations. For a more detailed explanation the reader is referred to the works of Olver [1], and Bluman and Kumei [2]. Lie symmetry methods deal with invertible point transformations of a differential equation, which map every solution of a differential equation to another solution. In section III we utilize this method to determine group transformations and reductions of the exponential Webster Horn equation to derive new solutions.

Given an equation of the form

$$
f(x)=0 \text {, }
$$

where $x=\left(x_{1}, x_{2}, \ldots x_{n}\right) \in \mathbb{R}^{n}$, and $f$ is a differentiable function. We consider a set of point transformations $x^{*}=X(x ; \varepsilon)$, depending on parameter $\varepsilon \in \mathbb{R}$. Expanding in the neighbourhood of $f(x)=0$ leads to

$$
x^{*}=x+\varepsilon X(x)+O\left(\varepsilon^{2}\right) \text {. }
$$

The transformation (5) is called the infinitesimal transformation of (4), where $X=\left(X_{1}, X_{2}, \ldots X_{n}\right)$ is given by $\left.X_{i}=\frac{\partial X_{i}}{\partial x}(x ; \varepsilon)\right)_{\varepsilon=0} 1 \leq i \leq n$ and the quantities $X_{i}$ are called the infinitesimals. Hence, given (5), solving the initial value problem $\frac{\partial x^{*}(x ; \varepsilon)}{\partial \varepsilon}=X(x), x^{*}(x, 0)=x$ recovers the oneparameter transformation group.

The infinitesimal operator of a one-parameter group, with infinitesimals $X_{i}$, is defined as the first order operator 
$V=X_{i}(x) \frac{\partial}{\partial x_{i}}$.

An equation of the form (4) is invariant under (5) if $f\left(x^{*}\right)=f(x)$. Hence substitution of (5) into (4) leads to a system of determining equations, which are linear partial differential equations (PDEs) in $X_{i}$. The most general infinitesimal operator $V$ is found by solving this system of PDEs. A function $G(x)$ is an invariant of (4) under (5) if $V G(x)$ $=0$. We form these invariants by solving the characteristic equation:

$$
\frac{d x_{1}}{X_{1}(x)}=\frac{d x_{2}}{X_{2}(x)}=\cdots=\frac{d x_{n}}{X_{n}(x)} \text {. }
$$

This determines a basis of functionally independent invariants for a group with operator $V$. Several algorithms and packages exist which determine the symmetries of systems of PDEs. This work uses the symmetry-determining package Dimsym [9] under Reduce [10].

\section{LIE GROUP ANALYSIS OF THE EXPONENTIAL WEBSTER HORN EQUATION}

Consider the exponential Webster Horn Equation (2), we seek a group transformation of the form:

$$
\begin{aligned}
x^{*} & =x+X(x, t, p) \varepsilon \\
t^{*} & =t+T(x, t, p) \varepsilon \\
p^{*} & =p+P(x, t, p) \varepsilon
\end{aligned}
$$

where $V=X(x, t, p) \frac{\partial}{\partial x}+T(x, t, p) \frac{\partial}{\partial t}+P(x, t, p) \frac{\partial}{\partial p}$ is the infinitesimal generator.

Using the symmetry-determining package Dimsym [9], the symmetries found were:

$$
\begin{aligned}
& V_{1}=\frac{\partial}{\partial t}, \quad V_{2}=p \frac{\partial}{\partial p}, \quad V_{3}=2 \frac{\partial}{\partial x}-m p \frac{\partial}{\partial p}, \\
& V_{4}=2 x \frac{\partial}{\partial t}+2 c^{2} t \frac{\partial}{\partial x}-c^{2} m t p \frac{\partial}{\partial p} .
\end{aligned}
$$

and the infinite symmetry: $V_{\infty}=\theta(x, t) \frac{\partial}{\partial p}$ where $\theta(x, t)$ is

any arbitrary solution to equation (2). The infinite symmetry arises due to the linearization of equation (2) and enables any linear combination of solutions to also be a solution.

While any symmetry may result in a solution to the governing equation (2), so too any linear combination of symmetries can lead to a solution. We seek to find the minimal set of linear combinations such that the maximal set of solutions is found. Following the method of commutators and adjoint maps, outlined in Olver [1], the minimal set is found to be: $\left\{V_{1}, V_{2}+\alpha_{1} V_{1}, V_{2}+\alpha_{3} V_{3} ; V_{3}, V_{4}+\alpha_{2} V_{2}\right\}$ where $\alpha_{i} \in \mathbb{R}$. We examine each element of the minimal set to establish our set of transformations and solutions.

Take, for example, the element $V_{4}+\alpha_{2} V_{2}$ and set $\alpha_{2}=0$.
Solving the initial value problems:

$$
\begin{aligned}
& \frac{\partial x^{*}(x, t, p, \varepsilon)}{\partial \varepsilon}=2 c^{2} t,\left.x^{*}\right|_{\varepsilon=0}=x \\
& \frac{\partial t^{*}(x, t, p, \varepsilon)}{\partial \varepsilon}=2 x,\left.t^{*}\right|_{\varepsilon=0}=t \\
& \frac{\partial p^{*}(x, t, p, \varepsilon)}{\partial \varepsilon}=-c^{2} m t p,\left.p^{*}\right|_{\varepsilon=0}=p,
\end{aligned}
$$

results in the group transformation:

$$
\begin{aligned}
& x^{*}=x \cosh [2 c \varepsilon]+c t \sinh [2 c \varepsilon] \\
& t^{*}=t \cosh [2 c \varepsilon]+\frac{x}{c} \sinh [2 c \varepsilon] \\
& p^{*}=p \exp \left(-c^{2} m \varepsilon\left\{t \cosh [2 c \varepsilon]+\frac{x}{c} \sinh [2 c \varepsilon]\right\}\right) .
\end{aligned}
$$

The corresponding characteristic equation is:

$\frac{d x}{2 c^{2} t}=\frac{d t}{2 x}=\frac{d p}{-c^{2} m t p}$ with solution:

$\phi=x^{2}-c^{2} t^{2}, p=\mathrm{A}(\phi) e^{\frac{-m x}{2}}$.

Substitution into the governing equation (2) produces the reduced ordinary differential equation (ODE):

$$
4 \phi \frac{d^{2} \mathrm{~A}}{d \phi^{2}}+4 \frac{d \mathrm{~A}}{d \phi}-\left(\frac{m}{2}\right)^{2} \mathrm{~A}=0
$$

with solution:

$$
\mathrm{A}(\phi)=\alpha \text { BesselJ }\left(0, \frac{1}{2} \sqrt{-m^{2} \phi}\right)+\beta \text { BesselY }\left(0, \frac{1}{2} \sqrt{-m^{2} \phi}\right) .
$$

Where $\alpha, \beta \in \mathbb{R}$ and BesselJ, BesselY are Bessels functions of the first and second kind respectively. Since we require the solution to be finite we take $\beta=0$ and hence

$p=\alpha$ BesselJ $\left(0, \frac{1}{2} \sqrt{m^{2}\left(c^{2} t^{2}-x^{2}\right)}\right) e^{\frac{-m x}{2}}$ is a solution to equation (2).

Employing the same method for each of the elements of the minimal set of symmetry combinations results in the complete set of transformations and solutions. These results are summarized in Table I and Table $I$ respectively. It is clear that the time harmonic wave solution (3) can be recovered from the general solutions listed in Table II by selecting appropriate parameters. Take, for example, the solution listed for the generator $V_{3}$, by choosing $\beta=0$ and $\frac{\mathrm{cm}}{2}=\omega$ the solution (3) is returned.

\section{OBJECT RECOGNITION}

Solving the initial value problem for each of the elements of the minimal set of symmetry combinations results in the complete set of transformations as summarized in Table $\mathrm{I}$. Consider a signal $p$, which satisfies the equation (2) and the transformations (9). Under these transformations the governing equation (2) is invariant i.e. equation (2), will hold for the 
transformed variables (9). Hence $p_{n}=\alpha p_{i ;}^{*}+\beta$ where it is 'desired that $\alpha \approx 1$ and $\beta \approx 0$. Under the transformation (9) $\alpha \equiv \cosh (2 c \varepsilon)$ thus $\alpha \approx 1$ for small $\varepsilon$. Also $\beta \leq O(\varepsilon)$ hence $\beta \approx 0$ for small $\varepsilon$, and so $p_{t}=p_{i j}^{*}$ for any $p$ which satisfies (2).

To verify the invariance of a signal in a practical situation, $p_{n}$ was measured using a polynomial fit as outlined in [11]. This was performed before and after $p$ underwent transformation and the normalized mean squared error (NMSE), between the two measurements, was calculated. It was postulated that, for a signal which satisfies (2), the NMSE will be a minimum. For normalization and calibration purposes the Bessel solution generated from $V_{4}$ was tested, using $\varepsilon=\frac{1}{2000 c}$; the resulting NMSE was found to be 0.0044 , and this was then normalized to 1 to give a baseline for further experiments.

A variety of mono audio signals, as summarized in Table III, were then tested. The majority of the resultant NMSE were found to range from double to over thirty times larger than for the pure Bessel solution. The exceptions to this are for the sinusoidal solution and the timpani. This is reasonable as the sinusoidal solution is the solution generated from $V_{3}$ and the timpani has a Bessel function inherent in its description. Thus the method is limited to identifying signals with similar characteristics.

The Bessel solution was then added to the individual audio signals to create mixtures where the Bessel solution comprised from 10 to $50 \%$ of the mixture. The mixed signals were then tested pre and post transformation. The results are again summarized in Table III. It can be seen that, with an increasing percentage of the Bessel solution present in the mixture, the NMSE approaches that of the pure Bessel solution (in this case normalized, for convenience, to 1). For example the female speech signal starts with a NMSE 17.8 times that of the Bessel Solution but is reduced to 3.75 times with the addition of only a $10 \%$ contribution of the Bessel solution. Similarly, the clarinet, which starts out with a NMSE of 36.8 times the Bessel solution, reduces to near unity by the time the mixture contains $30 \%$ of the Bessel solution. Thus the NMSE is a clear indicator of the presence of the Bessel solution in the audio mixture.

\section{CONCLUSION/DISCUSSION}

The symmetry reductions and exact solutions of the Webster Horn Equation (1) using the classical Lie method of infinitesimals has been discussed. Detailed analysis of the particular case of the exponential horn has resulted in a complete set of group transformations and solutions. A new solution was found which contains an exponentially decaying Bessel function. The general solutions found were examined and it was established that the known time-harmonic wave solution (3) was embedded therein. The use of the group transformations as a tool for audio object recognition was also explored with results indicating that the decaying Bessel function solution provides a particularly useful insight into audio object recognition. Practical results were presented which indicate the group transformations offers a mechanism for coarse recognition of audio objects which satisfy the exponential Webster Horn Equation (2).

\section{ACKNOWLEDGMENT}

The authors would like to thank Philip Broadbridge, Joanna Goard and Maureen Edwards for their guidance and recommendations.

\section{REFERENCES}

[1] P. J. Olver, "Applications of Lie Group Analysis to Differential Equations," 2nd ed. Springer-Verlag, New York, 1986.

[2] G. W. Bluman, S. Kumei, "Symmetries and Differential Equations," Springer-Verlag, New York, 1989.

[3] L. Van Gool, P. Kempenaers, A. Oosterlinck, "Recognition and SemiDifferential Invariants," Proceedings CVPR '91., IEEE Computer Society Conference on Computer Vision and Pattem Recognition, 1991. pp 454-460. 3-6 June 1991.

[4] D. G. Amold, K. Sturtz, V. Velten, N. Nandhakumar, " DominantSubspace Invariants" IEEE Transactions on Partern Analysis and Machine Intelligence, Vol. 22, No. 7, July 2000

[5] D. T. Blackstock, "Fundamentals of Physical Acoustics," John Wiley \& Sons, New York, 2000.

[6] G. R. Putland, "Every One-Parameter Acoustic Field Obeys Webster's Horn Equation," Journal of Audio Engineering Society. Vol. 41, No. 6, pp 435-45I. June 1993.

[7] S. W. Rienstra, "The Webster Equation Revisited," AlAA 20022520. $8^{\text {th }}$ AIAAVCEAS Aerospace Conference. Breckenridge, Colorado. 17-19 June 2002.

[8] E. Eisner, "Complete Solutions of the Websters Horm Equation" Joumal of the Acoustical Society of America. Vol. 41, No. 4, pp 1126-1146. 1967.

[9] J Sherring, "Dimsym users manual" Maths. Dept., La Trobe University, Melbourne, 1993.

[10] A. C. Heam, J. P. Fitch, "Reduce Users Manual" Konrad-ZuseZentrum, Berlin, 1998.

[11] L. Rabiner, B-H Juang, " Fundamental of Speech Recognition" Prentice Hall, Inc. New Jersey, 1993.

TABIE I: TRANSFORMATIONS

\begin{tabular}{|l|l|}
\hline$V_{1}$ & $x^{*}=x ; t^{*}=t+\varepsilon ; p^{*}=p$ \\
\hline$V_{2}$ & $\begin{array}{l}\text { As for } V_{2}+a_{1} V_{1} \text { or } V_{2}+a_{3} V_{3} \\
\text { with } a_{i}=0 \text { throughout }\end{array}$ \\
\hline$V_{3}$ & $x^{*}=x+2 \varepsilon ; t^{*}=t ; p^{*}=p e^{-m \varepsilon^{*}}$ \\
\hline$V_{4}$ & As for $V_{4}+a_{2} V_{2}$ with $a_{2}=0$ throughout \\
\hline$V_{2}+a_{1} V_{1}$ & $x^{*}=x ; t^{*}=t+a_{1} \varepsilon ; p^{*}=p e^{\varepsilon}$ \\
\hline$V_{2}+a_{3} V_{3}$ & $x^{*}=x+2 a_{3} \varepsilon ; t^{*}=t ; p^{*}=p e^{\left(1-m a_{3}\right) \varepsilon}$ \\
\hline$V_{4}+a_{2} V_{2}$ & $\begin{array}{l}x^{*}=x \cosh (2 c \varepsilon)+c t \sinh (2 c \varepsilon) ; \\
t^{*}=t \cosh (2 c \varepsilon)+\frac{x}{c} \sinh (2 c \varepsilon) ; \\
p^{*}=p \exp \left[a_{2} \varepsilon-c^{2} m \varepsilon\left\{t \cosh (2 c \varepsilon)+\frac{x}{c} \sinh (2 c \varepsilon)\right\}\right]\end{array}$ \\
\hline
\end{tabular}


TABLE II: REDUCED ODES AND SOLUTIONS

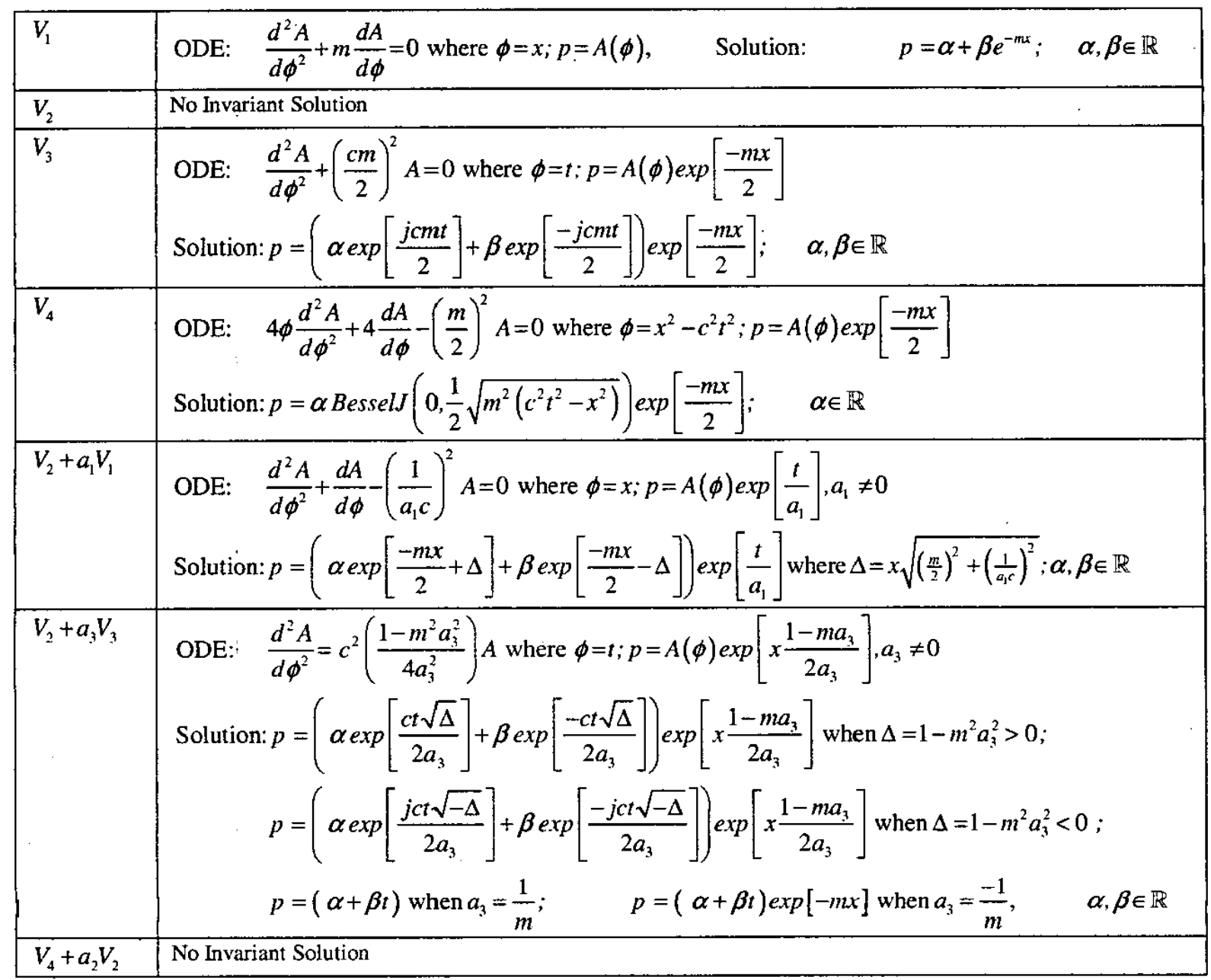

TABLE III: NORMALIZED MEAN SQUAREd ERROR FOR MiXEd AUdo SIGNAiS

\begin{tabular}{|c|c|c|c|c|c|c|}
\hline Sounds & $\begin{array}{l}\text { Individual } \\
\text { Sounds }\end{array}$ & \begin{tabular}{|c|} 
Mixt $10 \%$ \\
Bessel $+90 \%$ \\
Sound \\
\end{tabular} & $\begin{array}{c}\text { Mixt } 20 \% \\
\text { Bessel }+80 \% \\
\text { Sound }\end{array}$ & $\begin{array}{c}\text { Mixt } 30 \% \\
\text { Bessel }+70 \% \\
\text { Sound }\end{array}$ & $\begin{array}{c}\text { Mixt } 40 \% \\
\text { Bessel + } 60 \% \\
\text { Sound } \\
\end{array}$ & $\begin{array}{c}\text { Mixt } 50 \% \\
\text { Bessel + } 50 \% \\
\text { Sound }\end{array}$ \\
\hline 01 Bessel & 1.0000 & & & & & \\
\hline 02 Sinusoid Solution : & 1.2273 & 1.1818 & 1.1591 & 1.1364 & 1.1364 & 1.1136 \\
\hline 03 Timpani & 1.2727 & 1.1364 & 1.0455 & 1.0227 & 1.0000 & 0.9773 \\
\hline 04 Drum Mixture & 2.0455 & 2.0455 & 2.0227 & 2.0227 & 2.0227 & 1.5000 \\
\hline 05 Cello & 4.4545 & 1.6364 & 1.2500 & 1.0909 & 1.0000 & 0.9545 \\
\hline 06 Dulcimer & 2.2273 & 1.6591 & 1.1364 & 0.7955 & 0.7045 & 0.6818 \\
\hline 07 French Horn & 2.6591 & 1.5000 & 1.1818 & 1.0682 & 1.0227 & 1.0000 \\
\hline 08 Trumpet & 7.6591 & 3.1591 & 1.6136 & 1.1136 & 0.9091 & 0.7955 \\
\hline O9 Clarinet & 36.8636 & 7.9318 & 7.3182 & 1.3182 & 1.0682 & 0.9545 \\
\hline 10 Saxophone & 2.2045 & 1.3636 & 0.9318 & 0.6818 & 0.5682 & 0.5227 \\
\hline 11 Female Speech & 17.8636 & 3.7500 & 2.0000 & 1.5000 & 1.3182 & 1.2273 \\
\hline 12 Male Speech & 21.8409 & 20.0227 & 14.2273 & 1.1364 & 0.9545 & 0.8636 \\
\hline 13 White Noise & 2.7727 & 2.9545 & 1.4318 & 1.2045 & 1.0227 & 0.9545 \\
\hline
\end{tabular}

